\title{
Compatibility Assessment of Yoghurt Starters with Indigenous Isolates of Lactobacillus acidophilus for Development of Synbiotic Yoghurt by Checking Different Attributes like Contact Inhibition, Titrable Acidity, Viable Counts and pH
}

\author{
Suman Kandola* \\ Department of Biotechnology, Deenbandhu Chhotu Ram University of Sciences and \\ Technology (DCRUST), Murthal-132710, India \\ *Corresponding author
}

Keywords

Probiotics,

Prebiotics,

Synbiotics, Titrable

acidity, Viable

counts

Article Info

Accepted:

26 June 2018

Available Online:

10 July 2018

\section{A B S T R A C T}

Yogurt is produced by culturing with the lactic-acid producing bacteria Lactobacillus delbrueckii ssp. bulgaricus and Streptococcus thermophilus. Additional probiotic cultures can be added for the purpose of conferring their presumptive health benefits. Different starter strains Streptococcus thermophilus NCDC 74 (ST-74), Streptococcus thermophilus NCDC 311 (ST-311), Lactobacillus delbrueckii ssp. bulgaricus NCDC 9 (LB-9) and Lactobacillus delbrueckii ssp. bulgaricus NCDC 305 (LB-305) were comparatively evaluated for the compatibility with probiotics lactobacillus acidophilus NCDC 13 (LA13), Lactobacillus acidophilus NCDC 291(LA-291) to find out a desirable combination for the development of synbiotic yoghurt, by checking different attributes like contact inhibition, titrable acidity, viable counts and $\mathrm{pH}$. LA-13 shows more increment in the count during co-culturing with LA-291. Although LA-291 has more acid producing ability than LA-13, there is no considerable difference between the titrable acidity of these two. Further the increment in the count of LA-13 was higher with the combination LB-09 + ST74 than all other combinations. This study suggest the importance of incorporation of starter strains during preparation of synbiotic yoghurt with selected Lactobacillus acidophilus isolate as it increases the viability of probiotic strain during storage of food product and also improves the product characteristics.

\section{Introduction}

Probiotic cultures have been exploited extensively by the dairy industry as a tool for the development of novel products. Especially yogurt, are frequently used as probiotic delivery systems. According to the standards of identity listed in the United States Code of Federal Regulations (CFR), yogurt (in section
21 CFR part 131.200) is produced by culturing with the lactic-acid producing bacteria Lactobacillus delbrueckii ssp. bulgaricus and Streptococcus thermophilus. Additional cultures known as "probiotics" may be added for the purpose of conferring their presumptive health benefits. L. bulgaricus and $S$. thermophilus are used as the main yoghurt starters, and probiotics are added as 
supplements. Probiotics used in commercial products today are mainly members of the genera Lactobacillus and Bifidobacterium.

Some probiotics (especially lactic acid bacteria) are fastidious microorganisms, and are susceptible to environmental conditions, such as organic acid accumulation, high temperature, hyper osmotic pressure, low water activity, high redox potential (presence of oxygen) and the presence of inhibitory substances (Lourens-Hattingh and Viljoen, 2002). Consequently, survivability and stability of probiotics have become a marketing and technological challenge for the industry. Therefore, in order to obtain their desirable functional properties, probiotics need to be delivered in sufficient numbers with high viable rate (Talwalker and Kailasapathy, 2004). Yogurt starter cultures may enhance the growth and survival of probiotics by producing growth-promoting substrates or by reducing the oxygen content in milk (Saarela et al., 2000; Vinderola et al., 2002). S. thermophilus is a facultative anaerobic microorganism, which reduces the redox potential in milk by the consumption of oxygen by NADH oxidase (an enzyme catalyzed $\mathrm{NADH}+\mathrm{H}^{+}$with the presence of $\mathrm{O}_{2}$ to NAD and hydrogen peroxide) and pyruvate oxidase (an enzyme catalyzed pyruvate with the presence of $\mathrm{O}_{2}$ to acetate and $\mathrm{CO}_{2}$ ) (Teraguchi et al., 1978). Accordingly, this can create an anaerobic condition stimulating the growth of $L$. bulgaricus and probiotics in yoghurt (Lankaputhra and Shah, 1996). In addition, L. bulgaricus possess proteolytic enzymes that liberate amino acids such as valine, glycine, and histidine that are essential to some probiotics (lactobacilli species) (Tamime, 2005).

Since the efficacy of probiotics is related to the viable number at the time of consumption, they must survive during the processing, storage and transit through the gastrointestinal tract. Yoghurt starters possess some benefits during fermentation. Hence the aim of this study is to comparatively evaluate the compatibility of starter strains with the lactobacillus strains to find out a desirable combination for the development of synbiotic yoghurt.

\section{Materials and Methods}

\section{Bacterial strains and growth conditions}

The probiotic strains Lactobacillus acidophilus NCDC 13 (LA-13), Lactobacillus acidophilus NCDC 291(LA-291) and starter strains Streptococcus thermophilus NCDC 74 (ST-74), Streptococcus thermophilus NCDC 311 (ST-311), Lactobacillus delbrueckii ssp. bulgaricus NCDC 305 (LB-305) and Lactobacillus delbrueckii ssp. bulgaricus NCDC 09 (LB-09) used in the present investigation were obtained from the National Collection of Dairy Cultures (NCDC), Dairy Microbiology Division, National Dairy Research Institute (NDRI), Karnal, India. Freeze dried lactic cultures were activated in chalk litmus milk at $37^{\circ} \mathrm{C}$ for $24 \mathrm{hr}$. The yoghurt starters, Streptococcus thermophilus strains were activated and maintained in M17 medium (Himedia, Mumbai, India), while the Lactobacillus bulgaricus strains were activated and maintained in RCA medium (Himedia, Mumbai, India), and sub-cultured monthly. Before use, the lactic cultures were subcultured twice in de Man Rogosa Sharpe (MRS) broth (Himedia, Mumbai, India) and the yoghurt starters in their respective mediums.

\section{Compatibility of L. acidophilus strains with yoghurt starters}

The compatibility of probiotic L. acidophilus strains with yoghurt cultures was evaluated by well-diffusion agar assay and further confirmed by growth and activity studies by co-culturing in skim milk. 


\section{Well-diffusion agar assay}

The starter cultures whose inhibitory actions have to be determined were grown in $10 \%$ reconstituted skim milk at $37^{\circ} \mathrm{C}$ for $24 \mathrm{hr}$. Cell-Free Supernatants (CFS) were obtained by centrifugation at $3300 \mathrm{X} \mathrm{g}$ for $20 \mathrm{~min}$ at $5^{\circ} \mathrm{C}$. Sterilization of CFS was done by filtration through $0.45 \mu \mathrm{m}$ pore filter. Twenty millilitre of MRS agar (for L. acidophilus), M17 agar (for $S$. thermophilus) and RCA (for L. bulgaricus) was melted, tempered to $45^{\circ} \mathrm{C}$ and vigorously mixed with $200 \mu \mathrm{l}$ of an overnight culture and poured into petri dishes. After setting, wells of $5 \mathrm{~mm}$ in diameter were made and $50 \mu 1$ of CFS of the respective strain was placed into each well. The plates were kept in the refrigerator for proper diffusion and incubated aerobically or anaerobically at $37^{\circ} \mathrm{C}$ for $48 \mathrm{hr}$. After incubation, the plates were examined for clear zones around well. Experiments were replicated three times (Vinderola et al., 2002).

\section{Compatibility in relation to growth and activity}

The two probiotic L. acidophilus strains were inoculated in co-culture with two combinations of yoghurt starters as enlisted in table 1 . Sterlized $12 \%$ reconstituted skim milk was used in the medium for growth. The yoghurt starters were inoculated $(2 \%)$ in the ratio of $1: 1$ and probiotic $L$. acidophilus strains $(1 \%)$ were used as controls. All the samples were incubated at $42^{\circ} \mathrm{C}$ for $4 \mathrm{hr}$. The samples were analyzed for $\mathrm{pH}$, titrable acidity and individual counts before and after the incubation period.

\section{Analysis of samples}

\section{pH}

The $\mathrm{pH}$ of the samples was measured using a Digital pH meter (Model CP901, centaury) after calibrating with $\mathrm{pH} 4.0$ and $\mathrm{pH} 7.0$ standard buffers.

\section{Titrable acidity}

The acidity of the samples in terms of percent lactic acid was determined as per the method described in IS : 1479 (1960).

\section{Selective enumeration of organisms}

One $\mathrm{ml}$ of the sample was diluted with $9 \mathrm{ml}$ of $0.1 \%$ peptone water and mixed uniformly with a vortex mixture. Subsequent serial dilutions were prepared and viable numbers enumerated using pour plate technique. The counts of $S$. thermophilus were enumerated on M17 agar after incubation aerobically at $37^{\circ} \mathrm{C}$ for $48 \mathrm{hr}$ (Terzaghi and Sandine, 1975). RCA agar adjusted to $\mathrm{pH} 5.3$ and anaerobic incubation at $37^{\circ} \mathrm{C}$ for $72 \mathrm{hr}$ was used for the selective enumeration of L. bulgaricus (Dave and Shah, 1997 b). Maltose-MRS agar was used for the enumeration of L. acidophilus, as L. bulgaricus did not utilize maltose. Incubation was carried out anaerobically at $37^{\circ} \mathrm{C}$ for $48 \mathrm{hr}$ (Hull and Roberts, 1984).

\section{Results and Discussion}

Evaluation of compatibility of $L$. acidophilus strains with yoghurt starters

In order to select a suitable combination for the manufacture of yoghurt containing $L$. acidophilus, the interactions among the organisms was checked by well diffusion agar assay and the effect on the growth and acid production was studied by co-culturing them in skim milk.

\section{Well-diffusion agar assay}

A total of four strains of yoghurt starter bacteria (two of each $S$. thermophilus and $L$. bulgaricus) and two probiotic L. acidophilus 
strains were tested for interactions among them using cell-free supernatants (CFS) obtained from skim milk cultures. Four behaviours were observed with this methodology: complete inhibition (a clear absence of growth around the well), weak inhibition (partial inhibition around the well), absence of inhibition and more growth around the well.

\section{Effect of yoghurt starters on L. acidophilus strains}

The effect of CFS obtained from yoghurt starters on the growth of L. acidophilus strains is presented in table 2. As evident from the table, the Lb-09 completely inhibited the growth of LA-291, whereas ST-74 showed a partial inhibition of growth of LA-291. Both the yoghurt starters Lb-09 and ST-74 did not affected the growth of LA-13, which suggests its compatibility with these starters. It was found that the yoghurt starters Lb-305 and ST311 had no effect on the growth of both the probiotic strains. Vinderola et al., (2002) also observed a weak inhibition by L. bulgaricus supernatant on the growth of $L$. acidophilus strains.

Dave and Shah (1997a) suggested that the possible factors affecting the viability of $L$. acidophilus strains could be the antagonism by yoghurt strains or the higher concentration of hydrogen peroxide produced by L. bulgaricus. The partial inhibition of ST-74 on the probiotic strain LA-291 may be due to the higher acid production by the former organism.

\section{Effect of probiotic strains on yoghurt starters}

Table 3 shows the effect of cell-free supernatants of L. acidophilus strains on the growth of L. bulgaricus and S. thermophilus strains. The probiotic strain LA-291 showed a partial inhibition on the growth of LB-305, but had no effect on the growth LB-09, whereas the strain LA-13 showed absence of interaction towards both the $L$. bulgaricus strains. The cell-free supernatants of both probiotic strains had a stimulatory effect on the growth of $S$. thermophilus strains, as more growth was observed around the well. $L$. acidophilus was shown to produce bacteriocin against several strains of $L$. bulgaricus, $L$. helveticus etc. (Dave and Shah, 1997a). Vinderola et al., (2002) also reported a complete growth inhibition of L. Bulgaricus A61 when cultured in cell-free supernatant of L. Acidophilus CNRZ 1881. The growth stimulation of $S$. Thermophilus strains may be attributed to some growth factors produced by L. Acidophilus strains during the growth in skim milk for $24 \mathrm{hr}$.

\section{Compatibility in relation to growth and activity}

The growth and activity of probiotic strains in pure culture and in combination with yoghurt cultures was studied in order to select the more compatible cultures.

Growth compatibility of $L$. acidophilus strains with yoghurt starters LB-09 and ST74

The increment in count of the individual cultures, attained after co-culturing is depicted in table 4. The probiotic strain LA-291 recorded an increment in count by $1.85 \mathrm{log}$ cycles in culture, but the count decreased to the extent that it was not detectable during coculturing with yoghurt starters. The increase in the count of LA-13 following an incubation period of $4 \mathrm{hr}$ was $1.88 \mathrm{log}$ cycle in pure culture and 1.07 log cycle in combination. In general, the growth of $L$. acidophilus was found to decrease during co-culturing with yoghurt starters. This is in agreement with the result obtained by Oliveira et al., (2001), who 
reported that the mixed cultures resulted in lower counts of probiotic strains and it could be explained by a mechanism of nutritional competition.

The increment in count of L. bulgaricus did not show much difference in presence of $L$. acidophilus strains. The increase in count was $1.41 \log$ cycle with ST-74 and slightly declined to the extent of 1.10 to $1.30 \log$ cycle during co-culturing with $L$. acidophilus strains. On the contrary the counts of $S$. thermophilus showed a slight increase (1.52 to $1.75 \log$ cycle), while used in combination with probiotic strain, which confirm the synergistic action between them as observed in well-diffusion agar assay. The less growth of L. bulgaricus in presence of $L$. acidophilus strains can also be attributed to the completion of nutrients.

Figure 1 represents titrable acidity in terms of lactic acid after an incubation period of $4 \mathrm{hr}$ ranged from 0.31 to $0.43 \%$ in cases of pure cultures of L. acidophilus and 0.91 to $0.96 \%$ (Table 4) in case of combination which suggests that the L. acidophilus is the slow acid producer as compared to yoghurt starters and require long incubation period. The $\mathrm{pH}$ also showed similar pattern with the lowest value (4.51-4.63) in case of combination when compared to pure cultures (6.16-6.33). Similar results have been reported by Oliviera et al., (2001). The time needed to attain the maximum acidification rate was found to be longer for pure cultures of L. acidophilus than that with mixture cultures. Shah (2001) reported that it was necessary to add yoghurt starters to attain the desired characteristics in the finished probiotic yoghurt.

\section{Growth compatibility of $L$. acidophilus strains with yoghurt starters LB-305 and ST-311}

Table 5 presents the growth and activity of $L$. acidophilus strains during co-culturing with yoghurt starter combination LB-305 and ST311. As evident from the data, the increment in the count of LA-291 declined to $0.70 \mathrm{log}$ cycle, while that of LA-13 declined to $0.73 \mathrm{log}$ cycle during co-culture. This again suggests that co-culturing reduces the count of $L$. acidophilus (Oliviera et al., 2001). The increment in the count of LB-305 found to be lowest $(1.21 \log \mathrm{cfu} / \mathrm{ml})$ when co-cultured with LA-291. This confirms the result obtained in well-diffusion agar assay that LB305 was partially inhibited by LA-291. There was no significant difference in the increment in count of LB-305 in presence of NCDC 13. Co-culturing with $L$. acidophilus did not exhibit much difference in the count of ST311.

Table.1 The combinations of yoghurt starters and probiotic L. acidophilus strains used for compatibility studies

\begin{tabular}{|c|c|c|c|c|}
\hline $\begin{array}{l}\text { Sl. } \\
\text { No. }\end{array}$ & Yoghurt Starters & $\begin{array}{c}\text { Probiotic } L \text {. acidophilus } \\
\text { Strains }\end{array}$ & $\begin{array}{c}\text { Incubation } \\
\text { Temperature }\left({ }^{\circ} \mathrm{C}\right)\end{array}$ & $\begin{array}{c}\text { Incubation } \\
\text { Time (hr) }\end{array}$ \\
\hline \multirow[t]{2}{*}{1.} & ST-NCDC $74(1 \%)$ & \multirow[t]{2}{*}{ LA-NCDC 291 (1\%) } & \multirow[t]{2}{*}{42} & \multirow[t]{2}{*}{4} \\
\hline & LB-NCDC $09(1 \%)$ & & & \\
\hline \multirow[t]{2}{*}{2.} & ST-NCDC $74(1 \%)$ & \multirow[t]{2}{*}{ LA-NCDC $13(1 \%)$} & \multirow[t]{2}{*}{42} & \multirow[t]{2}{*}{4} \\
\hline & LB-NCDC 09 (1\%) & & & \\
\hline \multirow[t]{2}{*}{3.} & ST-NCDC $311(1 \%)$ & \multirow[t]{2}{*}{ LA-NCDC 291 (1\%) } & \multirow[t]{2}{*}{42} & \multirow[t]{2}{*}{4} \\
\hline & LB-NCDC 305 (1\%) & & & \\
\hline \multirow[t]{2}{*}{4.} & ST-NCDC $311(1 \%)$ & \multirow[t]{2}{*}{ LA-NCDC $13(1 \%)$} & \multirow[t]{2}{*}{42} & \multirow[t]{2}{*}{4} \\
\hline & LB-NCDC 305 (1\%) & & & \\
\hline
\end{tabular}


Table.2 Effect of cell-free supernatants of yoghurt starters on probiotic L. acidophilus strains

\begin{tabular}{|l|c|c|c|c|}
\hline \multirow{2}{*}{ Probiotic Strains } & \multicolumn{4}{|c|}{ Cell-Free Supernatants (CFS) } \\
\cline { 2 - 5 } & LB-09 & LB-305 & ST-74 & ST-311 \\
\hline LA-291 & $*$ & - & $\times$ & - \\
\hline LA-13 & - & - & - & - \\
\hline
\end{tabular}

* = Complete inhibition, $\mathrm{x}=$ Weak inhibition, $-=$ No effect.

Table.3 Effect of cell-free supernatants of probiotic L. acidophilus strains on yoghurt starters

\begin{tabular}{|c|c|c|}
\hline \multirow{2}{*}{ Yoghurt Starters } & \multicolumn{2}{|c|}{ Cell-Free Supernatants (CFS) } \\
\cline { 2 - 3 } & LA-291 & LA-13 \\
\hline LB-09 & - & - \\
\hline LB-305 & $\times$ & - \\
\hline ST-74 & + & + \\
\hline ST-311 & + & + \\
\hline
\end{tabular}

$+=$ More growth around well, $X=$ Weak inhibition, $-=$ No effect.

Table.4 Growth Compatibility of L. acidophilus Strains with Yoghurt Starters L. bulgaricus NCDC 09 and S. thermophilus NCDC 74

\begin{tabular}{|l|c|c|c|c|}
\hline \multicolumn{1}{|c|}{ Culture Combination } & \multicolumn{3}{|c|}{ Increment in count after incubation (log cfu/ml) } \\
\cline { 2 - 5 } & LB-09 & ST-74 & LA-291 & LA-13 \\
\hline LA- 291 $(1 \%)$ & - & - & 1.85 & - \\
\hline LA- 13 $(1 \%)$ & - & - & - & 1.88 \\
\hline LB- 09 $(1 \%)+$ ST- 74 $(1 \%)$ & 1.41 & 1.52 & - & - \\
\hline LB- 09 $(1 \%)+$ ST- 74 (1\%)+ & 1.30 & 1.75 & ND & - \\
\hline LA- 291 $(1 \%)$ & & & & 1.09 \\
\hline $\begin{array}{l}\text { LB- 09 }(1 \%)+\text { ST- 74 (1\%)+ } \\
\text { LA- 13 }(1 \%)\end{array}$ & 1.10 & 1.73 & - & \\
\hline
\end{tabular}

$\mathrm{ND}=$ Not Determined

Table.5 Growth Compatibility of L. acidophilus Strains with Yoghurt Starters L. bulgaricus NCDC 305 and S. thermophilus NCDC 311

\begin{tabular}{|l|c|c|c|c|}
\hline Culture Combination & LB-305 & ST-311 & LA-291 & LA-13 \\
\hline LA-291 (1\%) & - & - & 1.94 & - \\
\hline LA-13 (1\%) & - & - & - & 1.82 \\
\hline LB-305 (1\%)+ ST-311 (1\%) & 1.52 & 2.16 & - & - \\
\hline $\begin{array}{l}\text { LB-305 (1\%)+ ST-311 (1\%) } \\
+ \text { LA-291 (1\%) }\end{array}$ & 1.21 & 1.95 & 0.70 & - \\
\hline $\begin{array}{l}\text { LB-305 (1\%)+ ST-(1\%)+ } \\
\text { LA-13 (1\%) }\end{array}$ & 1.39 & 2.18 & - & 0.73 \\
\hline
\end{tabular}


Fig.1 Effects on Titrable Acidity \& pH in Different Culture Combinations. A= LA- 291 (1\%), $\mathrm{B}=\mathrm{LA}-13(1 \%), \mathrm{C}=\mathrm{LB}-09(1 \%)+\mathrm{ST}-74(1 \%), \mathrm{D}=\mathrm{LB}-09(1 \%)+\mathrm{ST}-74(1 \%)+\mathrm{LA}-291$ $(1 \%), \mathrm{E}=\mathrm{LB}-09(1 \%)+\mathrm{ST}-74(1 \%)+\mathrm{LA}-13(1 \%)$

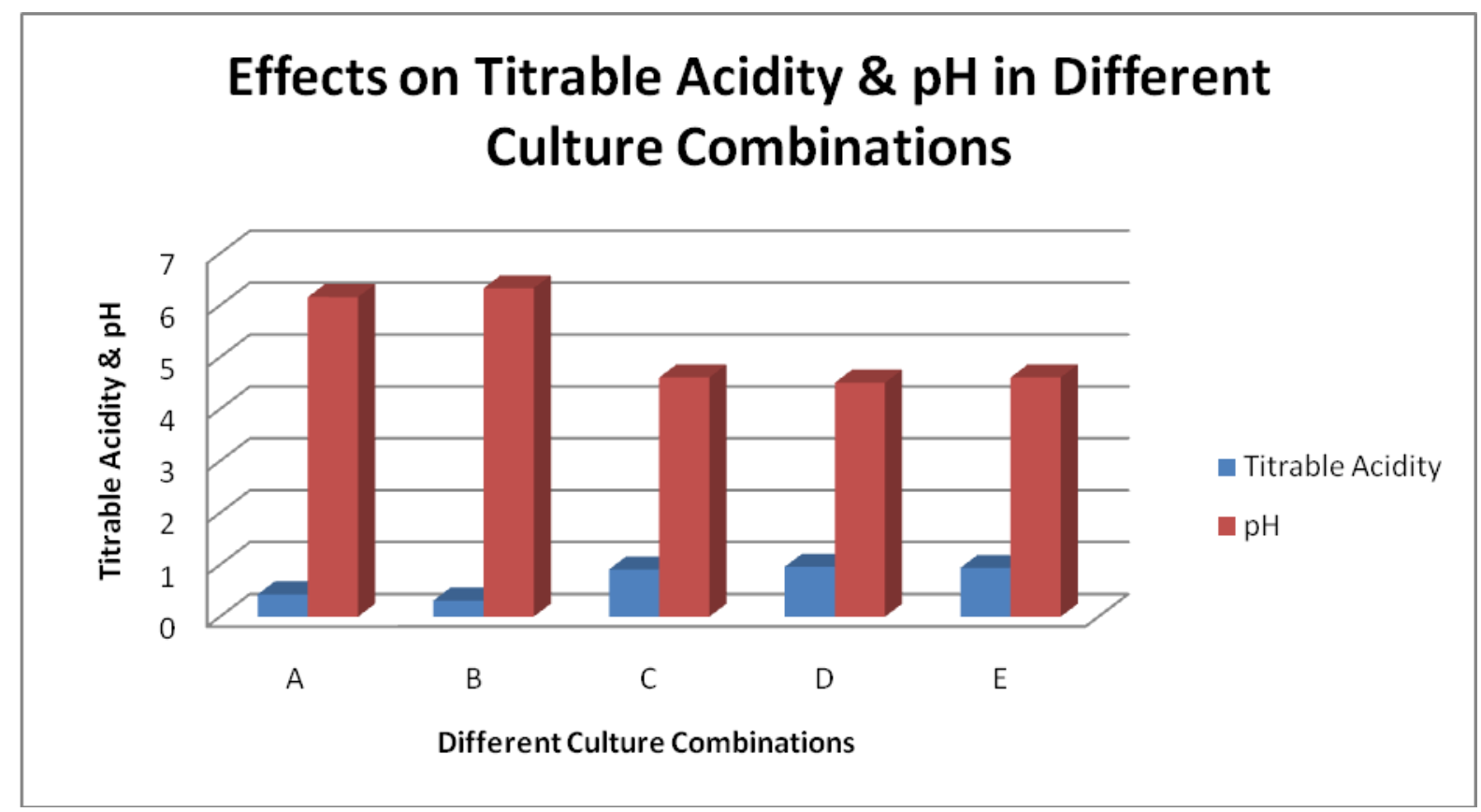

Fig.2 Effects on titrable acidity \& pH in different culture combinations. A= LA- $291(1 \%), \mathrm{B}=$ LA- $13(1 \%), \mathrm{F}=\mathrm{LB}-305(1 \%)+\mathrm{ST}-311(1 \%), \mathrm{G}=\mathrm{LB}-305(1 \%)+\mathrm{ST}-311(1 \%)+\mathrm{LA}-291$ $(1 \%), \mathrm{H}=\mathrm{LB}-305(1 \%)+\mathrm{ST}-311(1 \%)+\mathrm{LA}-13(1 \%)$

\section{Effects on Titrable Acidity \& pH in Different Culture Combinations}



Different Culture Combinations 
Figure 2 represents titrable acidity was found to be varying from $0.30-0.45 \%$ lactic acid in case of pure cultures and from 0.89 to $1.09 \%$ lactic acid in case of culture combinations. The highest titrable acidity was recorded for the combination of NCDC $305+311+291$. The $\mathrm{pH}$ of the fermented samples also showed similar trends for the lowest $\mathrm{pH}$ (4.41) for the culture combination containing LA-291. Among the pure culture also, LA-291 produced more acidity than LA-13. These results showed that LA-291 is a better acid producer as compared to LA-13.

This study concludes as follows:

\section{Selection of suitable combination}

The compatibility studies showed that $L$. acidophilus NCDC 13 is more compatible than L. acidophilus NCDC 291. Although LA-291 has more acid producing ability than LA-13, there is no considerable difference between the titrable acidity of these two. Therefore, with respect to the increment in the count during co-culturing LA-13 was selected as the probiotic strain for further studies. Further the increment in the count of LA-13 was higher $(1.09 \log \mathrm{cfu} / \mathrm{ml})$ with the combination LB-09 + ST-74. Also LB-09+ ST-74 are the defined and the commonly used yoghurt starters. Therefore the starter culture combination LB-09+ ST-74 and $L$. acidophilus NCDC 13 was selected for yoghurt making.

\section{Acknowledgment}

The CSIR (Council of Scientific and Industrial Research), New Delhi, India was acknowledged for financial assistance to first author. Author is also thankful to Dairy Microbiology Division, National Dairy Research Institute, Karnal, India, for providing the bacterial strains and the facilities for carrying out the work.
Abbreviations: CFU, Colony Forming Units

\section{References}

Dave, R.I. and Shah, N. P. (1997b). Viability of yoghurt and probiotic bacteria in yoghurt made from commercial starter cultures International Dairy Journal, 7: 31-41.

Dave, R.I. and Shah, N.P. (1997a). Effect of level of starter culture on viability of yoghurt and probiotic bacteria in yoghurts. Food Australia, 49: 164-168.

Hull, R.R., and Roberts, A.V. (1984). Diff erential enumeration of Lactobacillus acidophilus in yogurt. The Australian Journal of Dairy Technology, 39:160-163.

Lankaphuthra, W. E. V. and Shah, N. P. (1996). A simple method for selective enumeration of Lactobacillus acidophilus in yogurt supplemented with L. acidophilus and Bifidobacterium spp. Milchwissenschaft, 51(8):446-451.

Lourens-Hattingh, A. and Viljoen, B.C. (2002). Survival of probiotic bacteria in South African commercial Bioyoghurt. South African Journal of Science, 98:298-300.

Oliveira, M. N., Sodini, I., Remeuf, F. and Corrieu, G. (2001). Effect of milk supplementation and culture composition on acidification, textural properties and microbiological stability of fermented milks containing probiotic bacteria. International Dairy Journal, 11 935-942.

Saarela, M., Mogensen, G. Fonden, G. Mättö, J. and Mattila-Sandholm, T. (2000). Probiotic bacteria: Safety, functional and technological properties. Journal of Biotechnology, 84:197-215.

Shah, N.P. (2001). "Functional foods from probiotics and prebiotics." Food Technology, 55(11):46-53. 
Talwalkar, A., Miller, C.W., Kailasapathy, K. and Nguyen, M.H. (2004). Effect of packaging materials and dissolved oxygen on the survival of probiotics bacteria in yoghurt. International Journal of Food Science and Technology, 39:605-611.

Tamime, A.Y., Saarela, M., Sondergaard, A.K., Mistry, V.V. and Shah, N. P. (2005). Production and maintenance of viability of probiotic microorganisms in dairy products. In: Probiotic Dairy Products. Tamime, A. Y. ed., Blackwell Publishing: Oxford. pp. 39-72.

Teraguchi, S., Uehara, M., Ogasa, K., and
Mitsuoka, T. (1978). Enumeration of Bifidobacteria in dairy products. Japanese Journal of Bacteriology, 33: 753-761.

Terzaghi, B.E., and Sandine, W.E. (1975). Improved medium for lactic streptococci and their bacteriophages. Applied Microbiology, 29(6): 807813.

Vinderola, C.G., Mocchiutti, P. and Reinheimer, J.A. (2002). Interaction among lactic acid starter and probiotic bacteria used for fermented dairy products. Journal of Dairy Science, 85:721-729.

\section{How to cite this article:}

Suman Kandola. 2018. Compatibility Assessment of Yoghurt Starters with Indigenous Isolates of Lactobacillus acidophilus for Development of Synbiotic Yoghurt by Checking Different Attributes like Contact Inhibition, Titrable Acidity, Viable Counts and pH. Int.J.Curr.Microbiol.App.Sci. 7(07): 3743-3751. doi: https://doi.org/10.20546/ijcmas.2018.707.433 